\title{
Modes de communication entre médecins, mais pas seulement chez les Allemands
}

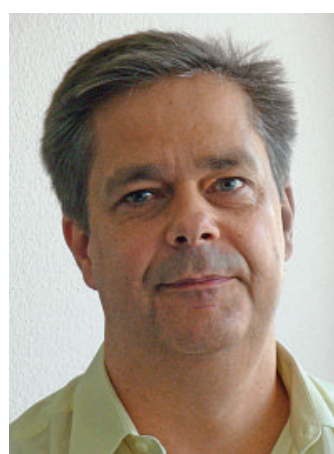

Eberhard Wolff
Comment rédiger la nécrologie d'un médecin-fonctionnaire décédé? Faire le résumé de sa vie? Parler du souvenir subjectif qu'on en garde? Dresser la liste de ses mérites?

Comment rédiger la nécrologie d'un médecin allemand, haut fonctionnaire, accusé d'avoir eu un passé nazi? La polémique suscitée par la nécrologie du Dr Hans Joachim Sewering, laquelle a omis la «part d'ombre» de sa biographie (cf. article à la page 1402), reflète les différents avis sur la question. Hormis le caractère politiquement explosif du sujet, cette affaire montre que la manière dont la société, et les médecins aussi, communiquent aujourd'hui change.

Il fut une époque où les médecins se présentaient volontiers comme des héros combattant pour alléger l'humanité de ses souffrances ou comme de bons samaritains se sacrifiant pour leur prochain. Et leurs nécrologies s'en faisaient l'écho. L'ancienne manière d'appréhender l'histoire de la médecine a également fortement contribué à promouvoir cette vision. Mais il fut également une époque où la règle voulait que les nécrologies ne mentionnent que le positif. Les médecins, du moins ceux qui étaient morts, n'étaient presque jamais montrés comme des êtres «normaux» ou faillibles (à l'exception néanmoins d'un Mengele). La nécrologie du Dr Sewering dans le Bulletin des médecins allemands s'inscrit dans cette tradition.

Certes, le principe du «De mortuis nil nisi bene» nous rappelle que les morts ne peuvent plus se défendre. Mais les nécrologies rigides vantant formellement les mérites d'une personne sont aujourd'hui mieux reconnues pour ce qu'elles sont: des textes très formels qui, à l'instar de nombreux discours du $1^{\text {er }}$ août, sont plus symboliques qu'informatifs. De tels éloges ne sont en général pas faits pour être discutés à coup de lettres de lecteur. Dans une société de communication comme la nôtre, les nécrologies rigides et traditionnelles sont de plus en plus considérées comme insuffisantes, en particulier si le mort a un tel passé.

Les autres réactions quant au décès du Dr Sewering illustrent parfaitement ce changement. Un auteur décrit, outre ses mérites, également les points sombres de son histoire et s'en distancie en n'utilisant aucun adjectif positif; une entorse prudente à la règle. Les textes formalisés offrent malheureusement peu de liberté, au même titre qu'un certificat de travail où la critique implicite ne peut s'exercer que par omission ou de manière codée.
Il n'est donc guère étonnant que les historiens de la médecine soient allergiques aux nécrologies à l'ancienne. Ils ne veulent plus jouer les applaudisseurs et encore moins les juges. Ils plaident au contraire pour une information historique approfondie sur les personnes décédées comme part intégrante de la société du savoir. Cette lettre de lecteur qu'ils défendent comme une «autre version des faits», n'est pas seulement le refus d'une science en mouvement qui cherche bien plus à analyser le passé qu'à le justifier ou à vanter les mérites d'un «grand médecin», mais aussi l'expression d'une communication plus ouverte au sein du corps médical.

Il y a longtemps que notre manière de parler en public se modifie. Au XVIII siècle déjà, les libres penseurs de la bourgeoisie européenne diffusaient un discours pour contrer les communications officielles des autorités, monarchiques pour la plupart. Le philosophe Jürgen Habermas avait déjà décrit ce phénomène en 1962 dans son livre «L'Espace public». Or, ce processus se heurte aujourd'hui aux derniers bastions de l'espace public formalisé.

La Suisse aussi a perdu il y a peu un des ses hauts fonctionnaires médecin: Hans Heinrich Brunner. Dans la nécrologie [1] publiée dans le Bulletin des médecins suisses, Ludwig T. Heuss parle non seulement d'une «personnalité exceptionnelle», d'un «titan» abattant un «travail surhumain», mais aussi des aspects contestés de sa vie, là où il avait ses limites et où il a failli. Ce portrait m'a touché par endroits, notamment quant au choix des photos qui montraient son côté humain. Ou s'agissait-il quand même d'un héros, mais d'un héros typiquement suisse?

Le débat soulevé par la nécrologie du Dr Sewering illustre, hormis l'explosivité du passé nazi, le clash de deux cultures de communication en grande partie dû aux mutations actuelles du paysage médiatique. Internet est utilisé comme un espace de communication plus informel. Le blog du journal des médecins allemands dit son fait au Dr Sewering et dans un autre blog libre, un médecin de campagne vide son sac quant à «l'arrogance» de Sewering envers les médecins de famille; ce qui a entraîné un internaute à écrire que, pour une fois, on est loin du «blabla nécrologique» d'usage (www.landarsch.blogger.de). Autres médias, autres mots.

Et vous, quelle nécrologie voulez-vous?

Eberhard Wolff* 